\title{
SUPRESSÃO DE CÉLULAS CANCERÍGENAS EM AUTÔMATO CELULAR
}

\section{SUPPRESSION OF CANCEROUS CELLS IN CELLULAR AUTOMATON}

\section{Kelly Cristiane Iarosz ${ }^{1}$, Robson Conrado Bonetti ${ }^{2}$, Antonio Marcos Batista ${ }^{3}$, Ricardo Luiz Viana ${ }^{4}$, Sergio Roberto Lopes ${ }^{5}$, Thadeu Josino Pereira Penna ${ }^{6}$}

1 Universidade Estadual de Ponta Grossa, Programa de Pós-graduação em Física $<$ kiarosz@gmail.com>.

${ }^{2}$ Universidade Estadual de Ponta Grossa, Programa de Pós-graduação em Física $<$ rcbonetti@gmail.com>.

${ }^{3}$ Universidade Estadual de Ponta Grossa, Departamento de Matemática e Estatística $<$ batista@interponta.com.br>.

${ }^{4}$ Universidade Federal do Paraná, Departamento de Física <viana@fisica.ufpr.br>.

${ }^{5}$ Universidade Federal do Paraná, Departamento de Física <lopes@fisica.ufpr.br>.

${ }^{6}$ Universidade Federal Fluminense, Centro de Estudos Gerais, Instituto de Ciências Exatas $<$ tjpp@if.uff.br $>$.

\section{RESUMO}

A cada ano, o câncer se torna uma das doenças com maior incidência na população mundial. Estimativas feitas pelo Instituto Nacional de Câncer mostram que, em 2010, cerca de 489.270 novos casos serão registrados somente no Brasil. Para auxiliar nos possíveis tratamentos, uma grande quantidade de pesquisas tem sido desenvolvida, abrindo respaldo para diversas tentativas de se obter modelos matemáticos que possam reproduzir acontecimentos orgânicos como a proliferação ocorrida na doença. Em geral, esses modelos mostram uma grande aproximação do que acontece, porém ressalta-se a dificuldade devida à complexidade dos mecanismos biológicos envolvidos. Usualmente, são feitas simplificações, a fim de encontrar soluções analíticas para as equações matemáticas propostas nos modelos. Apresentamos neste trabalho a proliferação do câncer por meio da infiltração de células cancerígenas em um tecido normal. Para tanto, foi utilizado um autômato celular que modela um sistema biológico, que é classificado em padrões espaço-temporais usando a distância de Hamming como diagnóstico. Com este diagnóstico, foi possível observar a supressão das células cancerígenas, variando os parâmetros do sistema.

Palavras-chave: Câncer. Distância de Hamming. Autômato celular. 


\begin{abstract}
The incidence of cancer has been increasing worldwide every year. Estimates made by the "Instituto Nacional do Câncer" show that in 2010, just in Brazil, about 489,270 new cases have been reported. In order to help possible treatments a lot of research has been developed. As new cases of cancer have increased, mathematical models became important as they can reproduce organic events such as the proliferation occurred in the disease. These models are generally very similar to experimental evidences, although they emphasize the difficulty to reproduce such events due to the complexity of the biological mechanisms involved. Simplifications are usually made to find analytical solutions to the mathematical equations proposed in the models. This study presents the growth of cancer considering the infiltration of cancer cells in normal tissues. It was used a simple cellular automaton that models a biological system, which is classified in spatio-temporal classes using the Hamming distance as a form of diagnosis. With this diagnosis it was possible to observe the suppression of cancerous cells, varying the system parameters.
\end{abstract}

Keywords: Cancer. Hamming distance. Cellular automaton.

\section{INTRODUÇÃO}

Nas últimas décadas, uma grande quantidade de experimentos tem sido voltada para o estudo de tumores cancerígenos. Também tem havido muitas tentativas de se obter um modelo matemático que represente mecanismos biológicos complexos. Foi estudado neste trabalho o crescimento do câncer utilizando um modelo de autômato celular bidimensional. O câncer é um dos grandes problemas da biologia matemática e tem levado diversos cientistas a formularem modelos com o propósito de analisar de que maneira a doença se prolifera. Além disso, a prevenção e o controle do câncer estão entre os mais importantes desafios científicos e de saúde pública da nossa época (BRASIL, 2010).

Os autômatos celulares são uma importante ferramenta computacional. Os primeiros trabalhos surgiram no final da década de 1940, pelo matemático John von Neumann (NEUMANN, 2002). O modelo é simples, porém se adéqua muito bem a sistemas naturais (WOLFRAM, 1982). É um modelo construído com base nos princípios evolutivos da natureza (BORRIES et al., 2007). As regras são as mesmas para todos os elementos de um sistema escolhido, e se derivam partindo de uma configuração inicial aleatória, que passa por uma evolução influenciando diretamente a vizinhança (WOLFRAM, 1986). É importante ressaltar que, embora as regras sejam as mesmas para todos os elementos do sistema, a situação dos componentes vizinhos pode variar indefinida e complexamente com o tempo, podendo originar novos sistemas e chegando até a sua autorreprodução (CASTRO; CASTRO, 2008).

Conforme suas características, os autômatos celulares podem ser distribuídos em classes I, II, III e IV, dependendo do comportamento em relação à evolução temporal (WOLFRAM, 1984, 2010).

Para avaliar a estabilidade do autômato celular, podendo assim verificar o processo de supressão das células cancerígenas, evolução do sistema foi comparada com o seu padrão inicial. Para esta comparação, utilizou-se a distância de Hamming (HAMMING, 1950), originalmente criada para detectar e corrigir erros na comunicação digital (WOLFRAM, 1983).

Assim, o trabalho foi organizado da seguinte forma: na seção 2, é feita uma introdução sobre autômato celular, conceitos básicos e suas implicações; na seção 3, é reportado o modelo de autômato celular aplicado na proliferação de câncer e os resultados obtidos utilizando como diagnóstico a distância de Hamming; e finalmente, na seção 4, as conclusões. 


\section{AUTÔMATO CELULAR}

Os autômatos celulares são modelos matemáticos discretos no espaço, no tempo e nas variáveis dinâmicas, sendo que sua evolução tem como princípio regras simples. São formados por unidades simples que interagem entre si e, à medida que o sistema evolui dinamicamente, emergem comportamentos complexos decorrentes dessas influências mútuas, o que é uma característica importante dos sistemas complexos (SILVA; MARTINS, 2003). Nos autômatos, são inseridas regras conforme o sistema que se deseja simular. Estas regras dependem do estado da própria célula e das células vizinhas e podem ser determinísticas ou probabilísticas. No entanto, os estados são definidos e finitos.

$\mathrm{Na}$ Figura 1 são apresentadas quatro regras com diferentes padrões representando a evolução temporal de um autômato celular. Para todas as regras foi disposta a semente em um mesmo valor, mas cada um dos padrões é obtido por meio de uma regra bem definida. No caso da Figura 1a, a regra utilizada é a 222, correspondente a 1101111 da representação binária de Wolfram (1986). Esta representação permite verificar como será a evolução do autômato no próximo passo de tempo. Na Figura $1 \mathrm{~b}$ apresentamos a regra 190, com sua representação binária dada por 10111110. Na Figura 1c temos a regra 90 - 01011010. E na Figura 1d, a representação da regra 30 - 00011110. Na evolução temporal, consideramos o 0 como sendo as posições denotadas em branco e o 1, em preto.

Na Figura 1 é possível observar o comportamento em relação à evolução temporal e separar em classes de acordo com o padrão apresentado. Identifica-se como classe I a Figura 1a, onde essa evolução mostra-se de maneira homogênea. Na Figura 1b, o padrão pertence à classe II, pois, conforme a evolução acontece, um conjunto de estruturas estáveis ou periódicas vai surgindo, diferentemente da Figura 1a, onde todas as posições apresentam valor igual a 1. Na Figura 1c, a classe encontrada é a III, com o autômato alcançando posições cada vez mais distantes, com variações de 0 e 1 formando padrões idênticos, porém com dimensões distintas. A Figura $1 \mathrm{~d}$ mostra a classe IV, na qual ocorre a formação de estruturas complexas ao longo da evolução temporal (WOLFRAM, 1984, 2010).
Podemos distinguir as classes de Wolfram utilizando a distância de Hamming. Para um autômato celular unidimensional, a distância de Hamming é dada pela seguinte relação:

$$
d_{H}(t)=\frac{1}{N}\left[N-\sum_{i}^{N} \delta_{\sigma_{i}(t-1) \beta_{i}(t)}\right]
$$

onde $\sigma_{\mathrm{i}}(t-1)$ e $\beta_{\mathrm{i}}(t)$ são os estados dos sítios $i$ em um tempo $t$, da configuração original e da réplica, respectivamente. $\mathrm{O}$ símbolo $\delta_{x y}$ é o delta de Kronecker, que é igual a 1 quando $x=y$ e nulo nos demais casos.

Figura 1 - Evolução temporal de autômatos celulares unidimensionais considerando(a) regra 222, (b) regra 190, (c) regra 90 e (d) regra 30, com os pontos pretos representam as posições com valor 1 e os brancos, com valor 0
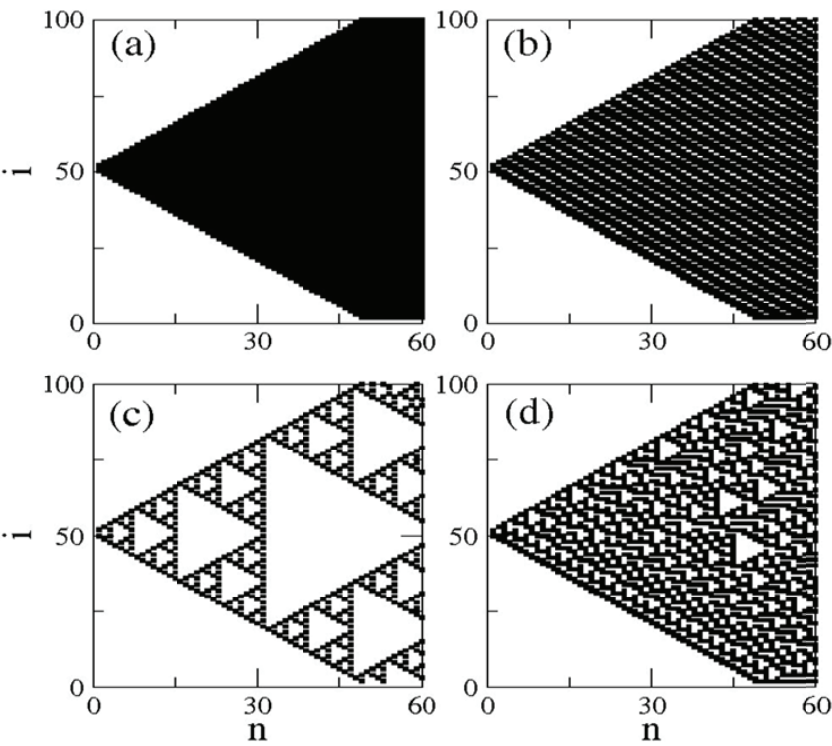

A Figura 2 mostra o cálculo da distância de Hamming considerando as mesmas regras utilizadas na Figura 1. Na Figura 2a, a curva cai abruptamente, o que está de acordo com o descrito na Figura 1a, classe I, onde o padrão observado na evolução temporal permanece igual ao padrão em um tempo anterior, e assim a distância de Hamming é nula. Na Figura 2b, a distância de Hamming passa a apresentar um valor fixo, após um transiente, com a diferença entre os padrões em $t-1$ e $t$ sendo constante, acordando com a Figura 1b, que apresenta regiões com estruturas periódicas ao longo da evolução, 
mas não perde sua homogeneidade. Na Figura 2c, a distância de Hamming apresenta oscilações irregulares, o que representa uma grande diferença entre os padrões no decorrer do tempo. Finalmente, na Figura 2d, a distância de Hamming apresenta um transiente irregular até chegar a um valor baixo e constante, o que é uma característica da classe IV (Figura 1d).

Figura 2 - Distância de Hamming para autômatos celulares unidimensionais considerando (a) regra 222, (b) regra 190, (c) regra 90 e (d) regra 30
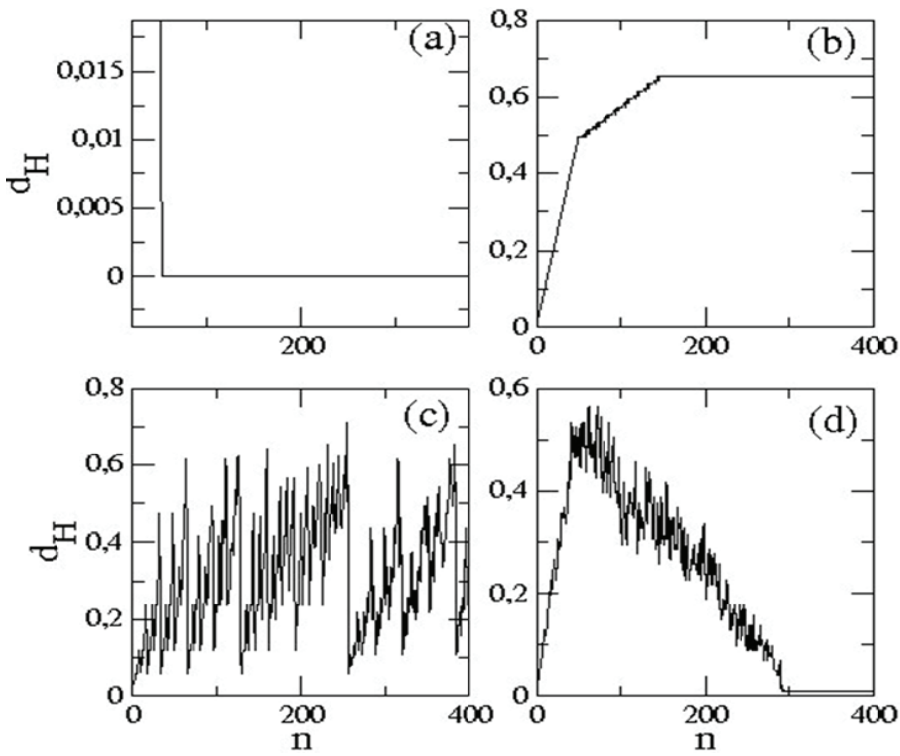

Com a distância de Hamming, foi estudada a supressão de células cancerígenas em um modelo de autômato celular bidimensional, observando a evolução temporal dos padrões de um tumor e comparando-o com um padrão que não apresenta tumor.

\section{PROLIFERAÇÃO DO CÂNCER}

Estudou-se um modelo de autômato celular proposto por Qi e colaboradores (1993) para analisarmos o crescimento do câncer. As células foram usadas como elemento principal, podendo ser cancerígenas, cancerígenas mortas, citotóxicas e células resultantes de processos citotóxicos, sendo denotadas por $C, D, E_{0}$ e $E$, respectivamente. As células citotóxicas - por exemplo, os glóbulos brancos - são capazes de eliminar tumores. Para isso podem ocorrer as seguintes reações:

$$
\begin{aligned}
& C \stackrel{k_{1}^{\prime}}{\longrightarrow} 2 C, \\
& C+E_{0} \stackrel{k_{2}}{\longrightarrow} E \stackrel{k_{3}}{\longrightarrow} E_{0}+D, \\
& D \stackrel{k_{4}}{\longrightarrow} .
\end{aligned}
$$

A reação 2 descreve a proliferação de células com câncer, com

$$
k_{1}^{\prime}=k_{1}\left(1-\frac{N_{C}}{\phi}\right)
$$

onde $k_{1}$ descreve a taxa de proliferação de células com câncer, $N_{C}$ é o número total de células cancerígenas e $\varnothing$ é uma constante, e assim $N_{C}$ atinge o máximo $ø$.

A primeira reação na equação 3 denota os processos citotóxicos, enquanto a segunda reação descreve a dissolução de complexos. A equação 4 descreve a dissolução de células mortas. Neste modelo, também é levada em consideração a densidade das células cancerígenas. Esta densidade $r$ descreve o efeito da pressão mecânica no desenvolvimento do câncer, e é dada por

$$
\rho=\frac{N^{\prime}}{R^{2}},
$$

onde $N^{\prime}=N_{\mathrm{C}}+N_{\mathrm{E}}+N_{\mathrm{D}}, \mathrm{R}=\left(\sum \mathrm{R}_{\mathrm{ij}}\right) / N^{\prime}$ e $R_{i j}$ representa a distância entre o sítio $(i, j)$ ocupado por uma célula cancerígena até a origem. Considerando um valor crítico de $\rho(t)$ quando $\rho=\rho_{C}$, ocorre que, se $\rho \leq \rho_{\mathrm{C}}$, a segunda célula filha resultante da proliferação poderá ocupar somente um dos sítios vizinhos mais interiores, que eram ocupados anteriormente por uma célula normal, como mostra a Figura 3, onde as células em cinza são as que pode ser ocupadas por uma célula filha. Se $\rho(t)>\rho_{\mathrm{C}}$, a segunda célula filha poderá invadir sítios vizinhos mais afastados da célula normal, que também eram ocupados anteriormente por células normais. Dessa forma, foi usada a mesma probabilidade de a segunda filha ocupar um dos dois possíveis sítios vizinhos.

Os valores adotados para os parâmetros $k_{1}$, $k_{2}, k_{3}$ e $k_{4}$ são listados na Tabela 1. Porém, a falta 
de dados experimentais para $k_{4}$ nos leva a considerar um valor compreendido dentro de um intervalo entre 0,1 e 0,4 (QI et al., 1993).

Figura 3 - O número 0 indica o centro da rede quadrada, onde I, II, III e IV representam os quatro quadrantes, e quando $\rho(t) \leq \rho_{\mathrm{C}}$, a segunda célula filha irá ocupar um dos dois sítios sombreados com uma probabilidade igual

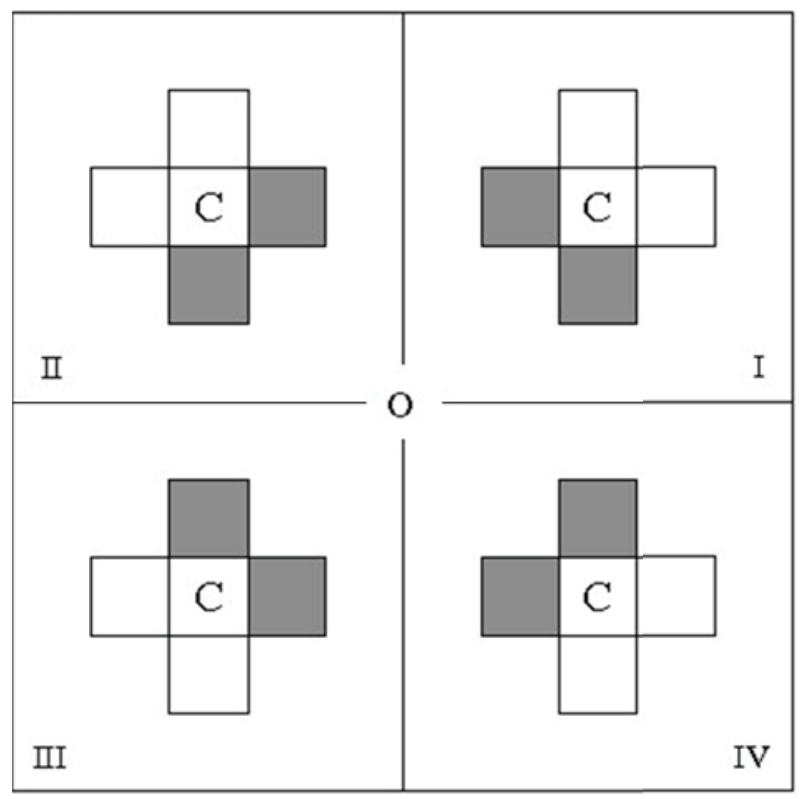

Tabela 1 - Valores adotados para os parâmetros $\mathrm{k}_{1}, \mathrm{k}_{2}, \mathrm{k}_{3}$ $\mathrm{e}_{4}$ (QI et al., 1993)

\begin{tabular}{ccc}
\hline Parâmetro & Valor & Tipos de células \\
\hline$k_{1}$ & $0,26-0,48$ & $\begin{array}{c}\text { Carcinoma espontâneo em } \\
\text { ratos do tipo C3H }\end{array}$ \\
\hline$k_{1}$ & $0,58-0,89$ & $\begin{array}{c}\text { Carcinoma do tipo KHT em } \\
\text { ratos }\end{array}$ \\
\hline$k_{2}$ & $0,2-0,4$ & $\begin{array}{c}\text { Macrófagos singenicamente } \\
\text { ativados }\end{array}$ \\
\hline$k_{3}$ & $0,2-0,65$ & $\begin{array}{c}\text { Macrófagos singenicamente } \\
\text { ativados }\end{array}$ \\
\hline$k_{4}$ & $0,1-0,4$ & \\
\hline
\end{tabular}

Quando consideramos os valores dos parâmetros dados por $k_{1}=0,68, k_{2}=0,4, k_{3}=k_{4}=0,4, \rho_{\mathrm{C}}=$ $3,85, \varnothing=10^{3}$ e $t=50$ a forma do tumor é obtida pela Figura $4 \mathrm{a}$. Em $t=0,5$, células cancerígenas estão na parte central da rede quadrada 101 x 101 e todos os outros sítios são ocupados por células normais, que são representadas em branco, sendo que em preto estão as células cancerígenas e em cinza os processos citotóxicos e as células mortas.

A Figura $4 \mathrm{~b}$ mostra a evolução temporal da quantidade de células cancerígenas, sendo que este comportamento segue o modelo de Gompertz (STELL, 1977), que geralmente é utilizado para descrever o crescimento cancerígeno e tem sua forma algébrica dada por

$$
V=V_{0} \exp \left\{\frac{A}{B}[1-\exp (-B t)]\right\},
$$

onde $V$ é o volume do tumor, $A$ e $B$ são parâmetros, $t$ é o tempo e $V_{0}$ é o volume inicial. Os parâmetros $A$ e $B$ são obtidos dos resultados experimentais utilizando-se o método dos mínimos quadrados. O modelo de Gompertz é simples e usual, porém, como se trata de um modelo puramente fenomenológico, não refletindo nenhum mecanismo microscópico do crescimento cancerígeno, houve a necessidade da utilização do autômato celular para um estudo microscópico do fenômeno. O modelo do autômato celular nos fornece uma explicação microscópica do modelo de Gompertz para o crescimento cancerígeno.

Figura 4 - (a) a forma de um tumor onde as células normais estão representadas em branco, sendo que em preto estão as células cancerígenas e em cinza os processos citotóxicos e as células mortas, onde i e j representam as posições das células

(b) a evolução temporal $n$ do número de células cancerígenas NC para $k_{1}=0,68, k_{2}=0,4$, $k_{3}=k_{4}=0,4, \rho_{\mathrm{C}}=3,85$
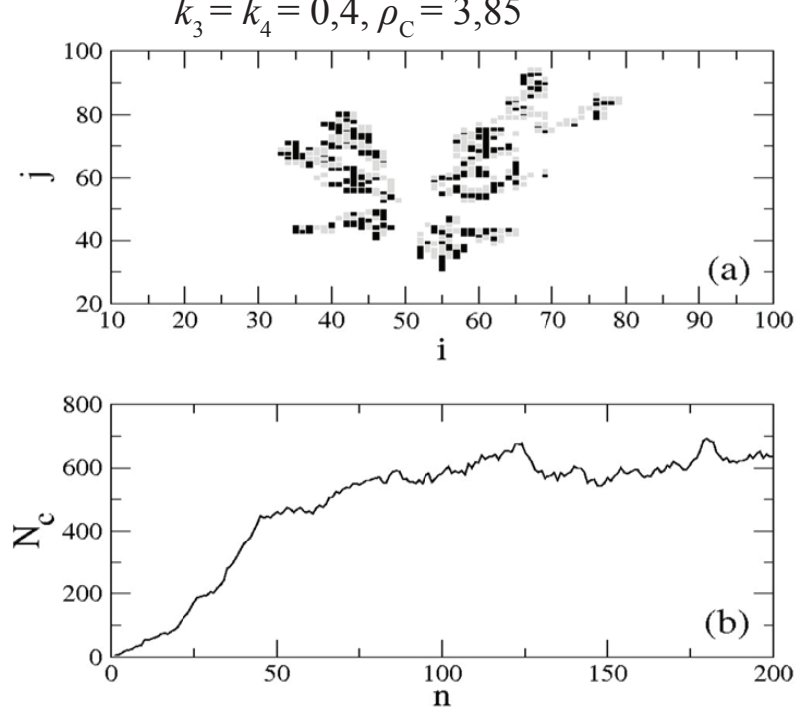
Uma técnica para estudar a estabilidade de autômatos celulares é comparar a evolução temporal do sistema original com a sua própria réplica, em que é inserida uma perturbação ou regras de evolução. Dessa forma, é possível utilizar como diagnóstico a distância de Hamming. Para um autômato celular bidimensional, a distância de Hamming é

$$
d_{H}(t)=\frac{1}{N^{2}}\left[N^{2}-\sum_{i, j}^{N^{2}} \delta_{\sigma_{i, j}(t) \beta_{i, j}(t)}\right],
$$

onde $\sigma_{i, j}(t)$ é o estado da célula $(i, j)$ no tempo $t$ e $\beta_{i, j}(t)$ é o estado da configuração original no tempo $t=0$. O símbolo $\delta_{x y}$ é o delta de Kronecker, que é igual a 1 quando $\mathrm{x}=\mathrm{y}$ e nulo nos demais casos.

A Figura 5 mostra a distância de Hamming calculada para as células cancerígenas em cada instante considerando uma rede $101 \mathrm{X} 101, k_{3}=k_{4}=0,4$, $\rho_{\mathrm{C}}=3,85, k_{2}=0,4$, (a) $k_{1}=0,68$ e (b) $k_{1}=0,60$. De acordo com as quatro classes de autômato celular, a Figura 5a corresponde à classe III pelo fato de a distância de Hamming crescer rapidamente, passando a apresentar um comportamento instável. Na Figura 5b, a distância de Hamming vai para um valor nulo com o passar do tempo, o que corresponde ao comportamento da classe I, que neste sistema está ocorrendo por causa da supressão das células cancerígenas.

Figura 5 - Evolução temporal da distância de Hamming considerando $N=101, k_{3}=k_{4}=0,4, \rho_{\mathrm{C}}=3,85$, $k_{2}=0,4$, (a) $k_{1}=0,68$ e (b) $k_{1}=0,60$
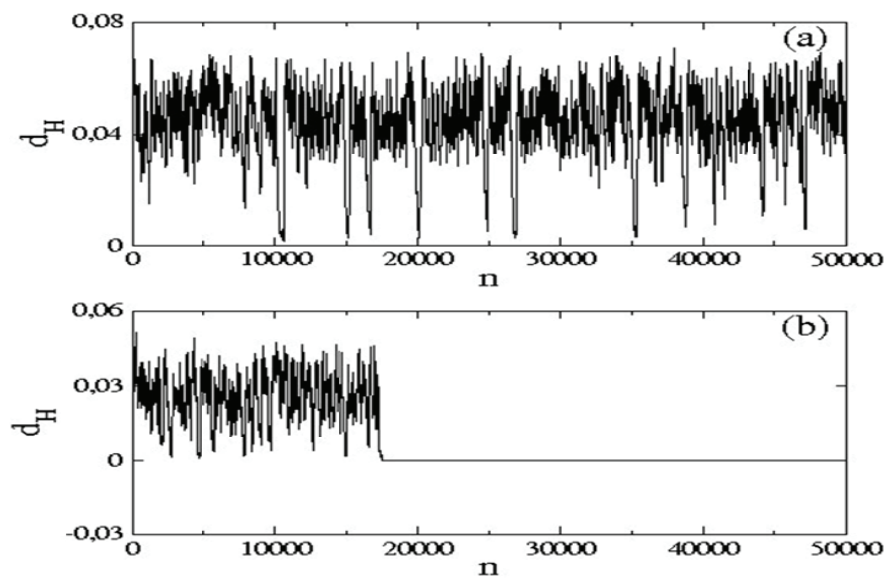

A distância de Hamming foi utilizada como diagnóstico para verificar a supressão ou não de células cancerígenas, porque no caso de supressão ocorre que $d_{H}=0$.

A região em cinza na Figura 6 denota os valores de $k_{1}$ e $k_{2}$ em que acontece a supressão de células cancerígenas. Pode-se verificar que para $k_{2}$ maior que 0,6 ocorre a supressão para qualquer valor de $k_{l}$, ou seja, não há o desenvolvimento do tumor, enquanto para $k_{2}$ menor que 0,46 o tumor irá sedesenvolver mesmo para valores baixos de $k_{l}$.

$\mathrm{Na}$ Figura 5b, percebe-se que após um transiente a distância de Hamming passa a apresentar valor nulo, sendo que o transiente depende dos valores de $k_{1}$ e $k_{2}$. Sendo assim, o tempo médio $n^{*}$ para que ocorra a supressão de células cancerígenas é

$$
n *=\frac{1}{K} \sum_{k}^{K} n_{k}^{\prime}
$$

onde $n_{k}^{\prime}$ é o tempo em que ocorre o transiente e $K$ o número de sementes utilizadas para iniciar o processo probabilístico da evolução do tumor. Fixando $k_{2}$ e variando $k_{1}$, de tal forma que os valores de $k_{1}$ estejam contidos na região cinza, conforme a Figura 6, verifica-se que o tempo médio aumenta com o aumento do valor de $k_{1}$. Também ocorre que, aumentando $k_{2}$ de 0,2 (Figura 7a) para 0,3 (Figura 7b), o maior valor atingido pelo tempo médio diminui, o que demonstra um tempo menor para a supressão de células cancerígenas.

Figura $6-k 1 \times k 2$, onde a região cinza corresponde a distância de Hamming nula

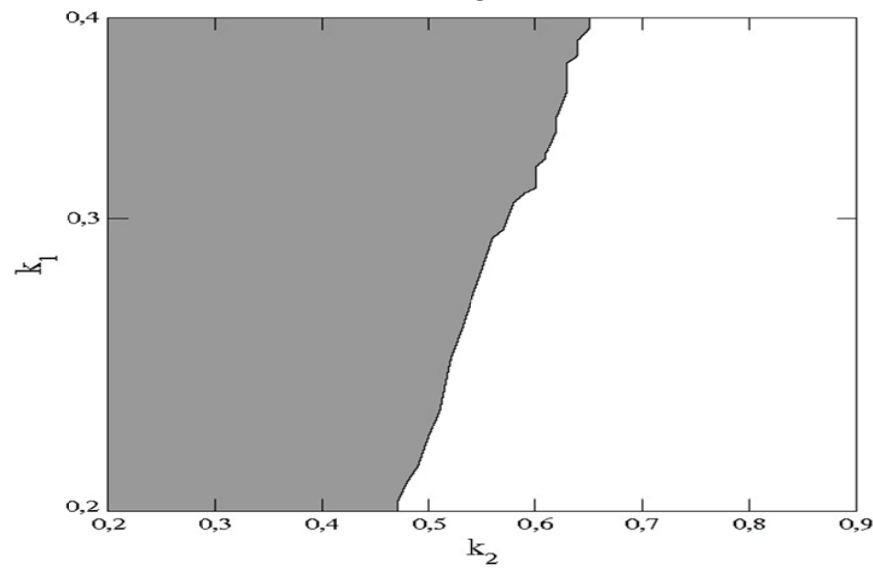


Figura 7 - Quando o valor de k1 aumenta, ocorre um aumento do tempo médio $\mathrm{n}^{*}$, sendo que foi considerado (a) $\mathrm{k} 2=0,2$ e (b) $\mathrm{k} 2=0,3$
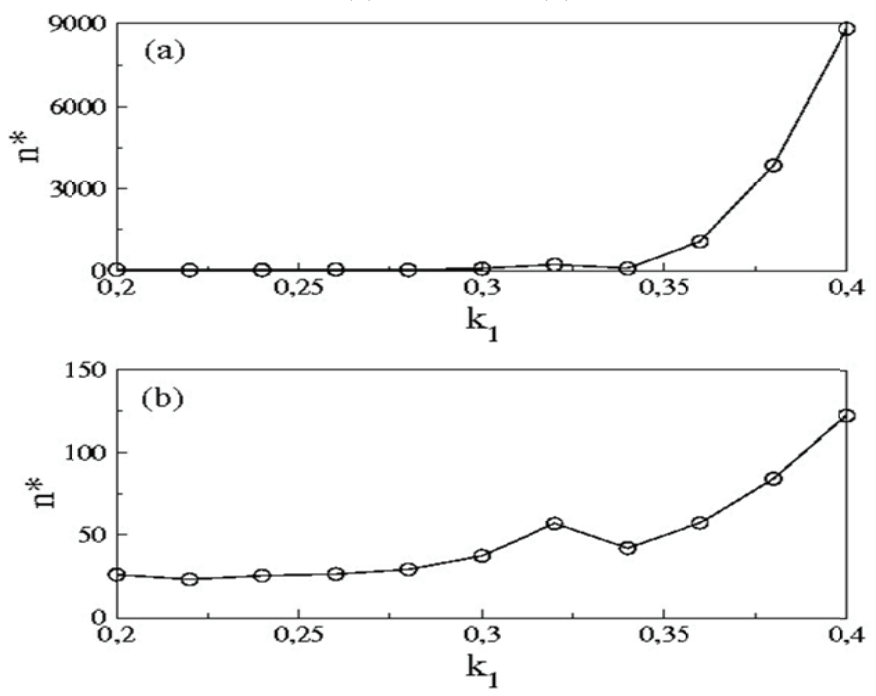

\section{CONCLUSÕES}

Neste trabalho, estudaram-se alguns aspectos da dinâmica do crescimento do câncer exibido por um autômato celular. Foi mostrado que a distância de Hamming pode ser usada como um diagnóstico da supressão de células cancerígenas. A supressão dessas células depende da taxa de proliferação de células com câncer e da taxa dos processos citotóxicos. O tempo médio para ocorrer a supressão aumenta com o aumento da taxa de proliferação e diminui com o aumento da taxa citotóxica.

\section{AGRADECIMENTOS}

A realização do trabalho foi possível devido ao auxílio financeiro das seguintes agências: CNPq, CAPES e Fundação Araucária.

\section{REFERÊNCIAS}

BORRIES, F. V.; WALZ, S. P.; BOETTGER, M. Space time play: computer games, architecture and urbanism: the next level. Boston: Birkhuser Architecture, 2007.

BRASIL. Ministério da Saúde. Instituto Nacional de Câncer. Inca lança estimativa 2010: incidência de câncer no Brasil. Disponível em: <http://www2.inca.gov.br>. Acesso em: 16 abr. 2010.
CASTRO, M. L. A.; CASTRO, R. O. Cellular automata's: Von Neumann, Conway and Wolfram's implementations. Revista de Ciências Exatas e Tecnologia, v.3, p.89-106, 2008.

HAMMING, R. W. Error decting and error correcting codes. The Bell System Technical Journal, v. 29, n. 2, p. 147-60, 1950.

NEUMANN, J. V. Theory of self-reproducing automata. Londres: UMI Reprint University of Illinois Press, 2002.

QI, A. S.; ZHENG, X.; DU, C. Y.; AN, B. S. A cellular automata model of cancerous growth. Journal of Theoretical Biology. v. 161, p. 1-12, 1993.

SILVA, H. S., MARTINS, M. L. A cellular automata model for cell differentiation. Physica A: Statistical Mechanics and its Applications, v. 322, p. 555-66, 2003.

STELL, G. G. Growth kinetics of tumors. Oxford: Clarendon Press, 1977.

WOLFRAM, S. Cellular automata as simple selforganizing systems. (1982) Disponível em: <www. stephenwolfram.com/publications/articles>. Acesso em: 04 maio 2010.

Cellular automata. (1983) Disponível em: $<w w w$. stephenwolfram.com/publications/articles $>$. Acesso em: 04 maio 2010 .

Statistical mechanics of cellular automata. Reviews of Modern Physics, v. 55, p. 601-44, 1983.

Universality and complexity in cellular automata. Physica D, v. 10, p. 1-35, 1984.

Theory and applications of cellular automata. Singapore: World Scientific Publishing, 1986. 